\title{
Using Race Model Violation to Explore Multisensory Responses in Older Adults: Enhanced Multisensory Integration or Slower Unisensory Processing?
}

DOI:

10.1163/22134808-00002588

\section{Document Version}

Accepted author manuscript

Link to publication record in Manchester Research Explorer

Citation for published version (APA):

Couth, S., Gowen, E., \& Poliakoff, E. (2017). Using Race Model Violation to Explore Multisensory Responses in Older Adults: Enhanced Multisensory Integration or Slower Unisensory Processing? Multisensory Research. https://doi.org/10.1163/22134808-00002588

Published in:

Multisensory Research

\section{Citing this paper}

Please note that where the full-text provided on Manchester Research Explorer is the Author Accepted Manuscript or Proof version this may differ from the final Published version. If citing, it is advised that you check and use the publisher's definitive version.

\section{General rights}

Copyright and moral rights for the publications made accessible in the Research Explorer are retained by the authors and/or other copyright owners and it is a condition of accessing publications that users recognise and abide by the legal requirements associated with these rights.

\section{Takedown policy}

If you believe that this document breaches copyright please refer to the University of Manchester's Takedown Procedures [http://man.ac.uk/04Y6Bo] or contact uml.scholarlycommunications@manchester.ac.uk providing relevant details, so we can investigate your claim.

\section{OPEN ACCESS}


1 Using Race Model Violation to Explore Multisensory Responses in Older

2 Adults: Enhanced Multisensory Integration or Slower Unisensory Processing?

3

4 Samuel Couth ${ }^{1}$, Emma Gowen $^{1}$ and Ellen Poliakoff ${ }^{1}$

$5{ }^{1}$ Faculty of Biology, Medicine and Health; The University of Manchester

6 Oxford Road

$7 \quad$ Manchester

8 UK

$9 \quad \mathrm{M} 13$ 9PL

10

11

12 Corresponding author: Samuel Couth

13 Samuel.couth@manchester.ac.uk

14 A3.05 Ellen Wilkinson Building

15 The University of Manchester

16 Oxford Road

17 Manchester

18 UK

$19 \mathrm{M} 13$ 9PL

20

21

22

23 
Older adults exhibit greater multisensory reaction time (RT) facilitation than young adults. Since older adults exhibit greater violation of the race model (i.e. cumulative distribution functions for multisensory RTs are greater than that of the summed unisensory RTs), this has been attributed to enhanced multisensory integration. Here we explored whether a) individual differences in RT distributions within each age group might drive this effect, and b) the race model is more likely to be violated if unisensory RTs are slower. Young $(n=34)$ and older adults $(n=30)$ made speeded responses to visual, auditory or tactile stimuli, or any combination of these (bi-/tri-modal). The test of the race model suggested greater audiovisual integration for older adults, but only when accounting for individual differences in RT distributions. Moreover, correlations in both age groups showed that slower unisensory RTs were associated with a greater degree of race model violation. Therefore, greater race model violation may be due to greater "room for improvement" from unisensory responses in older adults compared to young adults, and thus could falsely give the impression of enhanced multisensory integration.

\section{Keywords}


47 Our perception of the environment is based on the efficient interaction between multiple sensory signals (Stein and Meredith, 1993), which can be used to guide behaviour. Consistent with physiological studies showing increased neuronal activation in response to multisensory stimulation (Meredith and Stein, 1986), reaction time (RT) studies have shown faster responses for spatially and temporally coincident multisensory pairings compared to unisensory stimuli (for an early review see Nickerson, 1973). This multisensory response enhancement could be explained by detecting the fastest of either of the simultaneously presented unisensory stimuli (e.g. visual or auditory), or alternatively by neural integration of the bimodal stimuli (e.g. audiovisual). To infer which of these explanations is more likely, it is commonplace to compare the cumulative distribution function (CDF) of the multisensory RTs to the CDF of the unisensory RTs combined (i.e. the test of the race model; Miller, 1982; Miller and Ulrich, 2003; Colonius and Diederich, 2006; Ulrich, Miller and Schröter, 2007). Specifically, when two different sensory stimuli are presented simultaneously, the race model predicts that the unisensory signals are processed independently and in parallel to one another (Raab, 1962). The first unisensory signal to be detected (i.e. the "winner" of the race) determines the RT. The 'race model inequality' stipulates that the CDF for the bimodal RTs can never be greater than that predicted by the race model (Miller, 1982). If these conditions are met, then the race model is accepted (i.e. non-integration or statistical facilitation; Raab, 1962). On occasions where the bimodal CDF is greater, there is violation of the race model and so the multisensory response enhancement can be attributed to coactivation (i.e. multisensory integration) of the unisensory signals (Miller, 1982).

Given that increasing age is synonymous with decline in sensory organ function and unisensory cortical processing (Corso, 1971; Nusbaum, 1999; Correia et al., 2016) and that reduced white matter connectivity in older adults might affect long-range networks between sensory brain regions (Sullivan et al., 2001), it is reasonable to assume that the interaction between sensory signals would 
also be affected with increasing age (Poliakoff, Ashworth, et al., 2006; Poliakoff, Shore, et al., 2006). Surprisingly, however, this remains an under researched topic. Several experiments which have investigated the effects of ageing on multisensory integration (MSI) have utilised RT tasks. A consistent finding has emerged that older adults show a greater multisensory RT enhancement than young adults, which is evident for audiovisual (VA; Laurienti et al., 2006; Peiffer et al., 2007; Hugenschmidt, Mozolic and Laurienti, 2009) and visual-tactile (VT; Mahoney et al., 2011a) stimulus pairings. The test of the race model in these studies tends to show significant violation for older adults, suggesting that neural integration between the unisensory elements has occurred. Furthermore, compared to young adults, older adults show a broader range of RTs over which multisensory responses are greater than unisensory (Laurienti et al., 2006). Young adults also tend to show a reduced magnitude of race model violation, or perhaps no violation at all, thus suggesting minimal integration (e.g. Laurienti et al., 2006; Mahoney et al., 2011).

It has been suggested that increased multisensory facilitation in older adults may reflect a compensatory mechanism, whereby poorer acuity of the individual senses may lead to a greater degree of integration (Cienkowski and Carney, 2002; Laurienti et al., 2006; Dumas, Holtzer and Mahoney, 2016). These findings are consistent with the principle of inverse effectiveness, whereby MSI is more likely to occur and produce an enhanced response when combining two or more weak unisensory stimuli (Meredith and Stein, 1986; Stein and Meredith, 1993). Alternatively, older adults may be less capable of inhibiting irrelevant sensory stimuli (e.g. vision) when the task requires participants to attend to- and make a judgement about- a single modality (e.g. touch), and so information from different sensory modalities may be more readily combined (Poliakoff, Ashworth, et al., 2006; Poliakoff, Shore, et al., 2006; Couth, Gowen and Poliakoff, 2016). Whichever the explanation, it has been demonstrated that older adults show enhanced integration even in a simple reaction time task (Peiffer et al., 2007), thus it seems likely that this effect emerges early at the level of signal detection, prior to any higher-level processes which may be affected by general cognitive slowing in ageing (cf. Bucur et al., 2005; Laurienti et al., 2006). 
When investigating the effects of ageing on MSI, it is commonplace to compare young and older groups as a whole. Therefore, this makes previous experiments insensitive to individual differences in multisensory processing. In relation to RT experiments, it is uncertain whether the magnitude of race model violation might vary between different age groups, but only when taking into account individuals who show coactivation/integration. That is, older individuals who show robust integration of sensory cues (i.e. race model violation) could display even greater multisensory enhancements (i.e. an increased magnitude of violation) relative to young individuals who also show robust MSI. If this is the case, it would suggest that findings of enhanced MSI in older individuals are present, but not necessarily a universal finding among this population. Moreover, previous findings could be driven by the number of individuals within each age group who show race model violation.

Mahoney and colleagues (2014) recently addressed this issue by dividing participants into subgroups based on those who showed multisensory RT enhancement and those who did not. Participants whose RT facilitation to VT stimulation was in the lowest $25 \%$ (equating to an absolute multisensory RT enhancement of less than $8.1 \mathrm{~ms}$ ) were said to exhibit "No MSI effect", whilst those in the remaining $75 \%$ who showed a greater multisensory RT facilitation were said to exhibit a "MSI effect". Overall analysis failed to show significant race model violation. However, after sub-dividing the groups, those in the "MSI effect" group showed significant race model violation, whereas those in the "No MSI effect" group did not. These results demonstrate MSI in older adults, but only when considering individuals who show a RT benefit for multisensory stimuli. Given that there was no young group included in this study, it does not address whether young adults who show a "MSI effect" differ from older adults who also show this effect, or indeed whether the number of adults in each classification differs between age groups. The first aim of the current experiment, therefore, was to investigate whether enhanced MSI in older individuals is due to a greater number of older individuals who show race model violation and/or whether enhancement of MSI is accentuated in those older adults who show race model violation. In order to achieve this, we divided young and older participants into sub-groups based on individual race model performance. 
The current study also aimed to address the issue of ceiling or floor unisensory responses, which could provide an alternative explanation for enhanced MSI observed in older adults. Mahoney and colleagues (2014) noted that participants in their "No MSI" effect group did not appear to exhibit a multisensory benefit since they were already so fast to respond to one unisensory (usually tactile) stimulus (see also Barrett and Newell, 2015). The authors point out that these older adults might still be capable of MSI, however the visual stimulus was not facilitating a multisensory response. This could also be the case for young adults in previous experiments, where unisensory responses are already fast and at ceiling, thus the multisensory response can only be the same or slower (Holmes, 2009a, 2009b). In contrast, older adults tend to be slower to respond to unisensory stimuli compared to bimodal pairings (Diederich, Colonius and Schomburg, 2008). As such, older adults may show a greater magnitude of integration due to having a greater "room for improvement" from their unisensory RTs, or from an alternative perspective, fast unisensory responses may mask the potential to observe MSI in young adults. For example, if visual and auditory senses alone produce consistently slower RTs in older adults, the race model CDF formed from the summation of these unisensory elements will be less steep. As a result, the threshold for race model violation would be reduced for older adults, and so the magnitude of race model violation is likely to appear greater for the bimodal VA stimulus. Perhaps then, the amount of MSI shown by older adults compared to young may have been over-estimated in previous studies, or at least, it is not possible to infer enhanced multisensory integration in older adults from the test of the race model alone.

To address this issue, the current experiment compared bi- and tri-modal response enhancement (as assessed by tests of the race model for each individual) with unisensory performance. We predicted that individuals with the slowest unisensory reaction times (more likely to be older individuals) would show the greatest degree of race model violation, and thus give the appearance of enhanced MSI relative to those with faster unisensory responses i.e. a floor effect. 
Participants consisted of 34 young ( 20 female; mean age $=21.3 \pm 5.2$ ) and 30 older ( 16 female; mean age $=73.2 \pm 6.1)$ adults. Young adults consisted of psychology undergraduate students and other volunteers recruited via the University of Manchester's research volunteering website. Older adults were mainly recruited via local community groups and advertisements displayed in local newspapers and council webpages. All participants were initially screened to determine suitability for inclusion. All participants demonstrated equal to or better than 6/12 (20/40) visual acuity (with or without correction) assessed using a Snellen letter chart, indicating good acuity for all participants (Falkenstein et al., 2008; Kaiser, 2009). Auditory acuity was assessed using pure-tone audiometry to ensure that participants were able to hear the auditory stimulus used in the experiment; participants were required be able to hear $1000-2000 \mathrm{~Hz}$ at an intensity of $40 \mathrm{~dB} \mathrm{HL}$ in either ear. Older participants were screened for dementia using the Mini Mental Health Examination (MMSE; mean score $=29.3 \pm 1.0$. A score $\geq 24$ indicates normal cognition; Folstein, Folstein and McHugh, 1975). Participants had no history of any other neurological conditions (e.g. Parkinson's disease or neuropathy) or severe head injuries. Handedness was assessed via the Edinburgh Handedness Inventory (EHI; Oldfield, 1971. Right handed $n=27$ for both young and older adult groups). Participants received a small monetary reimbursement or course credits for taking part in this study. The study was approved by the University of Manchester Research Ethics Committee in accordance with the Declaration of Helsinki, and written informed consent was obtained before participation. Visual-tactile (VT), Audio-tactile (AT)], or trimodally [Visual-audio-tactile (VAT)]. Visual stimuli consisted of a single red LED flash $\left(\sim 3700 \mathrm{~cd} / \mathrm{m}^{2}\right)$ lasting for $50 \mathrm{~ms}$ (similar to Peiffer et al., 2007). The 
auditory stimulus consisted of a single $50 \mathrm{~ms}$ tone presented at $95 \mathrm{~dB} \mathrm{SPL}, 1300 \mathrm{~Hz}$, presented via a single Hi-TEX LX11 stereo speaker system positioned below the LED. The tactile stimulus consisted of

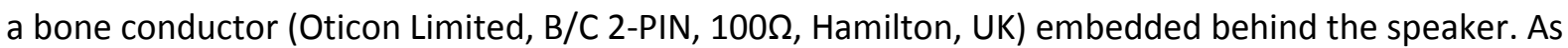
such, all three stimulus types were presented in close spatial proximity, thus appearing to have a common origin (Fig. 1). The pad of the participant's index finger was attached to the bone conductor using double-sided adhesive tape. The bone conductor was driven by sound files (white noise, $65 \mathrm{~dB}$ $\mathrm{SPL}, 3000 \mathrm{~Hz}$ ) from a PC through a Tacamp amplifier (Dancer Design, St. Helens UK) lasting for 50ms and was perceived as a tactile vibration. Bimodal and trimodal conditions consisted of perceptually synchronous (-30ms onset asynchrony for visual stimuli; see Hsiao, 1998) combinations of these stimulus types. Stimulus levels were clearly suprathreshold and kept constant for all participants.

Participants were also presented with continuous white noise via headphones (78dB SPL, $3000 \mathrm{~Hz})$ to eliminate any auditory cues produced by the tactile stimulus. Similarly, the outer casing of the $\mathrm{Hi}-$ TEX LX11 speaker was insulated using a sponge material to ensure that the auditory stimulus was not presenting any tactile cues to the hands (Fig. 1). Initial control experiments ( $n=5$; all young participants aged $<30$ ) were conducted to confirm that a) all stimuli were approximately iso-intense, b) the auditory stimulus was loud enough to be heard over the white noise presented via headphones, and c) to ensure that the auditory stimulus was not 
presenting any tactile cues to the participant's hands. These tests revealed that participants were largely unable to detect tactile cues produced by the auditory stimulus ( $95 \%$ undetected), whilst successfully detecting the auditory stimulus above the headphone white noise ( $99 \%$ detected). Participants reported all stimuli to be of equal intensity.

Procedure

Participants were seated in a darkened testing booth and adjusted their seating height so that their central gaze was in-line with the centre of the computer screen. Participants placed their index finger of their dominant hand on the bone conductor and pressed the toe of the corresponding foot down on a response pedal. Participants were then told that they would either see a red flash, hear a "beep", feel a vibration on their index finger, or any combination of the above. Participants were instructed to lift and replace their toe from the response pedal as soon as they noticed any type of stimulation. Participants were also instructed to fixate on the central fixation cross throughout the experiment and to avoid looking towards the stimuli.

Each trial began with a white central fixation cross $(19 \mathrm{~mm} \times 19 \mathrm{~mm} ; 4 \mathrm{~mm}$ bold) presented on black background screen for $750 \mathrm{~ms}$. The fixation cross then grew in size and boldness $(24 \mathrm{~mm} \times 24 \mathrm{~mm}$; $8 \mathrm{~mm}$ bold) and also changed to a silver colour for $750 \mathrm{~ms}$ to indicate the start of the trial. The fixation cross returned to its original size and colour and after a random delay of $500-1500 \mathrm{~ms}$ (to reduce temporal predictability) a stimulus was presented. The fixation cross remained onscreen throughout the trial. Making a response (toe lift) ended the trial and started the next. If participants responded prior to the onset of the stimulus a message displaying "Too early" was presented in the centre of the screen for $1500 \mathrm{~ms}$. A new trial automatically began after this error message. (280 trials total). Trials were broken up into four blocks of 70 and participants were given a short break between blocks. 
Error analysis and outlier removal

221 Errors in responding were classed as 'early' (pre stimulus onset), 'anticipatory' $(<100 \mathrm{~ms}$ post

222 stimulus onset) or 'late' (>1500ms post stimulus onset). Following the removal of these errors, outliers from each stimulus type, for each participant, were removed via the non-recursive procedure as described by van Selst and Jolicoeur (1994). For example, for a sample size of 40 trial repetitions per stimulus, RT values greater than 2.45 standard deviations from the mean RT were removed for each stimulus separately. The new mean RT without outliers was then calculated for each stimulus, for each participant. Mean errors were calculated separately for each age group.

Mean RT analysis

Although it is commonplace to compare mean RTs for each stimulus combination (VA, VT, AT and VAT) with their unisensory components ( $\mathrm{V}, \mathrm{A}$ and $\mathrm{T})$ to determine whether significant multisensory RT enhancements have occurred, that was not the focus in the current experiment. As such, no analysis of mean RTs was conducted. However, for completeness, mean RTs for each age group and for each sub-group were calculated and are displayed in Table 1.

Test of the race model - overall group comparisons

Each participant's RTs for each stimulus type were placed into ascending order. CDFs were then formed for each stimulus type by placing RTs into $5^{\text {th }}$ percentile bins. Each $5^{\text {th }}$ percentile bin was calculated by determining the range of RTs across the conditions in question (for example, for VA interactions, the fastest RT minus the slowest RT across V, A and VA conditions) and then multiplied by .05 . Accordingly, $5^{\text {th }}$ percentile bins were tailored for each individual and were specific to the conditions which were being analysed. This is especially important when comparing age groups, since a broader range of responses for older adults may produce the appearance of longer 
model prediction CDF was formed by taking the summed CDF of each unisensory component for a given multisensory condition, up until a value of 1 :

$$
\min \left[P\left(\mathrm{RT}_{\mathrm{X}} \leq t\right)+P\left(\mathrm{R} \mathrm{T}_{\mathrm{Y}} \leq t\right), 1\right]
$$

where $\mathrm{RT}_{\mathrm{x}}$ and $\mathrm{RT}_{\mathrm{y}}$ correspond to the RTs observed for sensory condition $\mathrm{X}$ (e.g. vision) and $\mathrm{Y}$ (e.g. auditory), for any time, $t$ (Colonius and Diederich, 2006).

Each participant's race model CDF was then subtracted from their multisensory CDF to generate a probability difference curve:

$$
R_{\mathrm{XY}}=P\left(\mathrm{RT}_{\mathrm{XY}} \leq t\right)-\min \left[P\left(\mathrm{RT}_{\mathrm{X}} \leq t\right)+P\left(\mathrm{RT}_{\mathrm{Y}} \leq t\right), 1\right]
$$

where $\mathrm{RT}_{\mathrm{XY}}$ corresponds to the RTs observed for the multisensory condition. Overall CDFs and difference curves were measured for each age group by taking the mean for all young and older adults separately (Fig. 2). To test whether race model violation was significant for each age group, one-sample $t$-tests were used to identify percentiles along the difference curve that were significantly greater than 0 ( $p<.0125$, Bonferroni correction). To avoid Type-1 error from conducting multiple $t$-tests, it has been suggested that $t$-tests should be limited to the $10^{\text {th }}-25^{\text {th }}$ percentile of the CDFs (i.e. $4 t$-tests), since this is where violation of the race model is most likely to occur (Kiesel, Miller and Ulrich, 2007).

To assess age related differences, independent samples $t$-tests were then conducted between the difference curves of young and older adults for the $10^{\text {th }}-25^{\text {th }}$ percentiles $(p<.0125$, Bonferroni correction; Fig. 2).

Test of the race model - sub-group analysis

Mahoney and colleagues (2014) formed their sub-groups based on individual multisensory RT facilitation (multisensory RT - fastest unisensory RT), with those in the lowest $25 \%$ of RT facilitation (equating to less than $8.1 \mathrm{~ms}$ ) in the "No MSI group", and the remaining $75 \%$ with greater RT 

increase in response to a bimodal stimulus could actually equate to a relatively large multisensory benefit. In comparison, sub-group divisions in the current experiment were determined based on the amount of race model violation for each individual, for each stimulus pairing. On occasions where an individuals' difference curve was $>0$ at any point between the $10^{\text {th }}$ and $25^{\text {th }}$ percentiles, they were placed into the "race model violation" (RMv) sub-group. Conversely, if an individual's difference curve was $\leq 0$ between the $10^{\text {th }}$ and $25^{\text {th }}$ percentiles, they were placed into the "race model acceptance" (RMa) sub-group. Mean CDFs and difference curves were then calculated separately for RMv and RMa sub-groups of both age groups. One-sample $t$-tests were used to identify percentiles where the RMv sub-group significantly violated the race model and age related differences were assessed using independent samples $t$-tests. Two-by-two (Sub-group; RMv vs. RMa, and Age group; Old vs. Young) chi-square tests were also conducted to determine whether the number of participants in each sub-group differed between each age group.

For each participant, the fastest unisensory response for a given multisensory condition (e.g. $\mathrm{V}$ or $\mathrm{A}$ for a VA stimulus combination) was selected and then group means were calculated for RMv and RMa sub-groups of both age groups, which were compared using independent samples $t$-tests. To gain further insight into whether multisensory enhancement was related to unisensory processing speed, correlations were performed between the magnitude of race model violation and each individual's fastest mean unisensory RT, for each age group separately.

Results 
290 young and older adults according to the non-recursive procedure (van Selst and Jolicoeur, 1994),

291 thus leaving $95.9 \%$ and $95.2 \%$ of data remaining for further analysis for young and older adults,

292 respectively. In general, outliers tended to be slower responses given that reaction time distributions

293 tend to be positively skewed, and that fast responses $<100 \mathrm{~ms}$ were excluded as anticipatory errors

294 prior to the non-recursive procedure. Given the high accuracy for both age groups and all conditions,

295 no further analysis was conducted.

296

297

298 
Table 1. Overall mean absolute RTs and mean absolute RTs for each age group for RMv and RMa sub-groups separately.

\begin{tabular}{|c|c|c|c|c|c|c|c|c|c|c|c|c|}
\hline \multicolumn{13}{|c|}{ Mean RTs (ms) } \\
\hline \multirow{3}{*}{$\begin{array}{l}\text { Stimul } \\
\text { us }\end{array}$} & \multicolumn{12}{|c|}{ RMv sub-group } \\
\hline & \multicolumn{3}{|l|}{ VA } & \multicolumn{3}{|l|}{ VT } & \multicolumn{3}{|l|}{ AT } & \multicolumn{3}{|l|}{ VAT } \\
\hline & $\begin{array}{l}\text { Overall }(n= \\
49)\end{array}$ & $\begin{array}{l}\text { Young ( } n= \\
27)\end{array}$ & $\begin{array}{l}\text { Older }(n= \\
22)\end{array}$ & $\begin{array}{l}\text { Overall }(n= \\
48)\end{array}$ & $\begin{array}{l}\text { Young ( } \mathrm{n}= \\
25)\end{array}$ & $\begin{array}{l}\text { Older }(n= \\
23)\end{array}$ & $\begin{array}{l}\text { Overall }(n= \\
51)\end{array}$ & $\begin{array}{l}\text { Young }(n= \\
26)\end{array}$ & $\begin{array}{l}\text { Older }(n= \\
25)\end{array}$ & $\begin{array}{l}\text { Overall }(n= \\
36)\end{array}$ & $\begin{array}{l}\text { Young }(n= \\
18)\end{array}$ & $\begin{array}{l}\text { Older }(n= \\
18)\end{array}$ \\
\hline $\mathrm{V}$ & $394(12)$ & $363(12)$ & $433(19)$ & $394(11)$ & $361(11)$ & $430(17)$ & - & - & - & $405(13)$ & $372(18)$ & $439(20)$ \\
\hline A & $368(12)$ & $343(11)$ & $398(21)$ & - & - & - & $358(11)$ & $339(11)$ & 379 (19) & $380(14)$ & $358(15)$ & $402(24)$ \\
\hline $\mathrm{T}$ & - & - & - & $388(11)$ & $364(8)$ & $415(20)$ & $377(11)$ & 355 (9) & 400 (19) & 400 (14) & $368(11)$ & $433(24)$ \\
\hline VA & $324(8)$ & $308(10)$ & $343(14)$ & - & - & - & - & - & - & - & - & - \\
\hline VT & - & - & - & 331 (9) & 314 (9) & $350(15)$ & - & - & - & - & - & - \\
\hline AT & - & - & - & - & - & - & $312(8)$ & $302(9)$ & $323(13)$ & - & - & - \\
\hline VAT & - & - & - & - & - & - & - & - & - & $322(10)$ & $312(11)$ & $331(16)$ \\
\hline Overall & $362(11)$ & $338(12)$ & $392(19)$ & $371(11)$ & $346(10)$ & 398 (19) & $349(11)$ & $332(11)$ & $367(18)$ & $377(14)$ & $352(14)$ & $401(23)$ \\
\hline \multirow{3}{*}{$\begin{array}{l}\text { Stimul } \\
\text { us }\end{array}$} & \multicolumn{12}{|c|}{ RMa sub-group } \\
\hline & \multicolumn{3}{|l|}{ VA } & \multicolumn{3}{|l|}{ VT } & \multicolumn{3}{|l|}{ AT } & \multicolumn{3}{|l|}{ VAT } \\
\hline & $\begin{array}{l}\text { Overall }(n= \\
15)\end{array}$ & $\begin{array}{l}\text { Young }(n= \\
7)\end{array}$ & $\begin{array}{l}\text { Older }(n= \\
8)\end{array}$ & $\begin{array}{l}\text { Overall }(n= \\
16)\end{array}$ & $\begin{array}{l}\text { Young ( } n= \\
\text { 9) }\end{array}$ & $\begin{array}{l}\text { Older }(n= \\
7)\end{array}$ & $\begin{array}{l}\text { Overall }(n= \\
13)\end{array}$ & $\begin{array}{l}\text { Young ( } n= \\
8 \text { ) }\end{array}$ & $\begin{array}{l}\text { Older }(n= \\
5)\end{array}$ & $\begin{array}{l}\text { Overall }(n= \\
28)\end{array}$ & $\begin{array}{l}\text { Young }(n= \\
16)\end{array}$ & $\begin{array}{l}\text { Older }(n= \\
12)\end{array}$ \\
\hline V & 371 (19) & $380(32)$ & $363(23)$ & $374(24)$ & $381(33)$ & $366(36)$ & - & - & - & $368(15)$ & $359(20)$ & $379(24)$ \\
\hline A & $328(21)$ & $345(40)$ & $313(21)$ & - & - & - & $359(26)$ & $357(36)$ & $362(44)$ & $331(13)$ & $327(18)$ & $336(20)$ \\
\hline $\mathrm{T}$ & - & - & - & 351 (19) & $342(23)$ & $363(34)$ & $388(23)$ & $369(24)$ & $418(47)$ & $351(10)$ & $347(13)$ & 357 (17) \\
\hline VA & $314(21)$ & $330(38)$ & $300(22)$ & - & - & - & - & - & - & - & - & - \\
\hline VT & - & - & - & $326(21)$ & $321(30)$ & $331(32)$ & - & - & - & - & - & - \\
\hline AT & - & - & - & - & - & - & $333(22)$ & 332 (29) & 335 (38) & - & - & - \\
\hline VAT & - & - & - & - & - & - & - & - & - & $295(12)$ & 295 (19) & 295 (14) \\
\hline Overall & $338(21)$ & $352(36)$ & $325(23)$ & $350(21)$ & 348 (29) & $353(33)$ & $360(24)$ & $353(29)$ & $372(43)$ & $336(14)$ & $332(18)$ & $342(21)$ \\
\hline
\end{tabular}

SEM is shown in parenthesis. These represent only the component mean RTs for each stimulus combination i.e. audiovisual condition $=\mathrm{V}, \mathrm{A}$ and $\mathrm{VA}$ only. 

For VA and VAT stimulus combinations, there were no significant race model violations between the $10^{\text {th }}-25^{\text {th }}$ percentiles for young or older adult groups (all $p>.0125$, Bonferroni correction). For the VT stimulus combination, there was significant race model violation at the $10^{\text {th }}$ percentile for the older adults [ $t(29)=2.754, p=.010]$, but no significant violation for the young adults at any percentile (all $p>.0125$, Bonferroni correction). Conversely, for the AT stimulus combination, young adults showed significant race model violation at the $10^{\text {th }}$ percentile $[t(32)=2.862, p=.007]$, whereas older adults showed a near significant violation only $[t(29)=2.620, p=.014]$. groups at any percentile, for any stimulus combination (all $p>.0125$, Bonferroni correction). 
325

326

327

328

329

330

331

332

333

[Fig. 2 Here]

334

335

336

337

338

339

340

341

342

343 
345

346

347

348

349

350

351

352

[Fig. 3 Here]

353

354

355

356

357

358

359

360

361 
The number of older and young individuals within each sub-group, for each combination is presented in Fig. 4. There were no significant differences in the number of people in each sub-group (RMv and RMa) between the age groups for any of the stimulus pairings (all $p>.05){ }^{1}$

367 The older RMv sub-group showed significant race model violation at the $10^{\text {th }}$ and $15^{\text {th }}$ percentiles, whilst the young RMv sub-group showed significant violation at the $10^{\text {th }}$ percentile only $(p<.0125$, Bonferroni correction). There was significantly higher race model violation for the older RMv subgroup compared to young at the $15^{\text {th }}$ percentile $[t(47)=2.715, p=.009]$, but no other significant differences between age groups at any other percentile ( $p>.0125$, Bonferroni correction).

The older RMv sub-group showed significant race model violation at the $10^{\text {th }}$ percentile only, whilst the young RMv sub-group showed significant violation at the $10^{\text {th }}$ and $15^{\text {th }}$ percentiles $(p<.0125$, Bonferroni correction). There were no significant differences between young and older RMv subgroups at any percentile (all $p>.0125$, Bonferroni correction).

\footnotetext{
${ }^{1}$ For the older adults, 11 participants were within the RMv sub-group across all multisensory conditions, whilst 12 participants were within the RMv sub-group for 3 out 4 of the multisensory conditions. Only one participant was within the RMa sub-group across all multisensory conditions, and only 5 participants were within the RMa sub-group for 3 out of 4 multisensory conditions. For the young adults, 12 participants were within the RMv sub-group across all multisensory conditions and 10 participants for 3 out 4 of the multisensory conditions. Six participants were in the RMa sub-group for 2 out of 4 multisensory conditions, and only 3 were within the RMa sub-group across all multisensory conditions. Therefore, the majority of participants were displaying race model violation, across the majority of multisensory conditions.
} 
378 Both older and young RMv sub-groups showed significant race model violation at the $10^{\text {th }}$ and $15^{\text {th }}$ 379 percentiles (both $p<.0125$, Bonferroni correction). There were no significant differences between 380 young and older RMv sub-groups at any percentile (all $p>.0125$, Bonferroni correction).

381 Visual-audio-tactile (VAT) combination

382 The older RMv sub-group showed significant race model violation at the $10^{\text {th }}$ percentile only $(p<$ 383.0125 , Bonferroni correction). The young RMv sub-group showed a near significant violation at the $38410^{\text {th }}$ percentile only ( $p=.014 ; p>.0125$ Bonferroni correction). Comparisons between the young and 385 older RMv sub-group also indicated a near significant difference at the $10^{\text {th }}$ percentile $[t(34)=2.476$, $386 p=.018]$, but no significant differences at any other percentile ( $p>.0125$, Bonferroni correction).

387 Race model acceptance analysis

Analysis of the RMa sub-groups did not show any significant difference between the young and older adults at any percentile, for any stimulus combination (all $p>.0125$, Bonferroni correction). 
391

392

393

394

395

396

397

398

[Fig. 4 Here] 
400

401

402

403

404

405

406

407

408

409

410

411

412

413

414

415

416

417

418

419

420

421

422

\section{5}

\section{6}

Given that significant differences in race model violation between the age groups was found for the VA stimulus only, we limit the analysis to this bimodal pairing. Prior to conducting this analysis, one young and one older participant with very slow unisensory RTs were removed using the nonrecursive procedure described by van Selst and Jolicoeur (1994) (see points to the right of where the data is clustered in Figs $5 b$ and $5 c$ ). Responses to the auditory stimulus were consistently the mean fastest of either $V$ or $A$, for both young $(n=27)$ and older $(n=22)$ participants. The fastest unisensory RT (i.e. either V or A) for the older RMv sub-group (374ms) was significantly slower than the older RMa sub-group (313ms) [ $t(27)=2.479, p=.020]$. In comparison, there was no significant difference between the young RMv sub-group $(336 \mathrm{~ms})$ and the young RMa sub-group (309ms) $[t$ $(31)=1.107, p=.227]$. When comparing between the age groups, the fastest unisensory RT was significantly slower for the older RMv sub-group than the young RMv sub-group $[t(46)=2.254, p=$ .029], but there was no significant difference between the older and young RMa sub-groups [ $t(12)=$ $.159, p=.876$ ] (Fig. 5a). The relationship between fastest unisensory RTs and the magnitude of VA race model violation showed a significant positive correlation for both older $[r=.480, \mathrm{n}=29, p=$ $0.008]$ and young $[r=.462, \mathrm{n}=33, p=.007]$ adults (Figs $5 \mathrm{~b}$ and $5 \mathrm{c}$ ). 
424 The aims of this study were twofold. First, the study aimed to investigate age differences in multisensory RT enhancement using an approach that takes individual differences into account, thus participants were divided into RMv and RMa sub-groups based on individual race model violations. Prior to sub-group divisions, there were no age differences in the magnitude of race model violation, suggesting no differences in integration. However, after sub-group divisions the older RMv subgroup showed significantly greater race model violation than the young RMv sub-group for the VA pairing only. As the number of participants in the older and young RMv group was similar, these results suggested that increased multisensory facilitation in older individuals is due to enhanced integration for certain older individuals. The second aim of this study was to determine whether the magnitude of race model violation was influenced by the speed of unisensory responses. Given that the older RMv sub-group's unisensory RTs were slower, plus there were significant positive correlations between the magnitude of race model violation and fastest unisensory responses, it is possible that this apparent enhancement of integration for older adults may be due to floor and ceiling effects for older and young adults, respectively.

Individual differences in the test of the race model

Analysis of race model violation prior to sub-group divisions produced findings which both support and contradict previous studies. Older adults exhibited significant race model violation for the VT pairing only; however this did not differ significantly from the young group. This contradicts most previous findings that older adults integrate more than young adults (Laurienti et al., 2006; Peiffer et al., 2007). In fact, the young adults displayed significant race model violation for the AT pairing, whilst older participants did not.

Following sub-group divisions, however, both young and older RMv sub-groups showed significant race model violation for all bimodal pairings. Consistent with previous research, older adults showed 
2006; Peiffer et al., 2007; Hugenschmidt, Mozolic and Laurienti, 2009). Consequently, through addressing the issue of individual differences within age groups, it was possible to observe an enhancement of race model violation (i.e. apparent multisensory integration) in older compared to young adults. Moreover, it was possible to rule out a higher number of individuals in the older RMv sub-group and/or a reduction in race model acceptance as a reason for this pattern of results.

Complementing the findings of Mahoney and colleagues (2011), the test of the race model for the AT and VT stimulus pairings did not show any difference between young and older RMv sub-groups. This suggests that, even when only accounting for individuals who show race model violation, there are no age-related differences in multisensory RT enhancements for AT and VT combinations. Moreover, the number of individuals and the magnitude of race model acceptance (i.e. nonintegration) did not differ significantly between the young and older RMa sub-groups. Accordingly, the finding that multisensory RT enhancements were equal between age-groups prior to sub-group divisions was not driven by different magnitudes of race model acceptance.

When examining the trimodal condition, the older RMv group showed significant violation, whereas the young RMv group did not. There have been numerous RT studies which have extended their stimulus set to include VAT combinations (Diederich and Colonius, 2004; Hecht, Reiner and Karni, 2008a, 2008b), however this is the first time that this has been explored in older adults. Lee, Poliakoff and Spence (2009) have previously examined how combinations of visual, auditory and tactile feedback from a touch screen device benefitted older participants' abilities to type in strings of numbers. Although the performance of older adults' was not improved in the trimodal combination in their study, participants reported that this condition was easier than all others. On the contrary, the current findings suggest that a sub-group of older adults continued to show a multisensory benefit with the addition of a third stimulus, whereas even those young adults who

471 showed race model violation reached a maximum response enhancement for bimodal stimuli. That 
472 is not to say that the older adults benefited more than the young adults, given that the difference 473 between the groups was non-significant.

474 Overall, it is possible that previous reports of enhanced (reduced) audiovisual integration in older 475 (young) adults may have been driven by individuals who show a greater magnitude of race model 476 violation (acceptance).

Enhanced multisensory integration - a matter of the fastest unisensory RTs?

Enhanced MSI in older adults, as inferred from greater violation of the race model, has previously been suggested to be a compensatory mechanism for poorer unisensory functioning (e.g. Laurienti et al., 2006; Dumas, Holtzer and Mahoney, 2016), and/or physical functioning (Mahoney, Holtzer and Verghese, 2014; Mahoney, Dumas and Holtzer, 2015; Teramoto et al., 2017). Others have also suggested that enhanced integration is due to a poorer ability to selectively attend to unisensory cues (Poliakoff, Ashworth, et al., 2006; Poliakoff, Shore, et al., 2006). Here, we propose an alternative explanation that the magnitude of MSI may not differ between age groups, at least for simple RT studies, but the methods and analysis technique used may give rise to- or the appearance of- enhanced integration in older adults.

Analysis of the fastest unisensory RTs for the VA stimulus combination demonstrated that the older RMv sub-group was significantly slower than the older RMa sub-group and the young RMv subgroup. Also, when the fastest unisensory RT was plotted against the magnitude of VA race model violation, it is clear that as unisensory responses slow down, the magnitude of violation increases, which is evident for both age groups. Consequently, it appears that the RMv sub-group - especially the older adults - show greater magnitude of violation due to having a greater "room for improvement" from their unisensory RTs. Therefore, there may not be an enhancement of integration in older adults compared to young, per se. Instead, it is possible that the threshold for race model violation is reduced for some older individuals due to having slower unisensory RTs, giving a biased impression of enhanced integration (i.e. a floor effect). From a different perspective, 
it may be harder to observe race model violation in young adults due to them having fast unisensory RTs, and thus gives the impression of reduced integration (i.e. a ceiling effect).

Given that this was a simple reaction test, it is unlikely that slower unisensory RTs in some older adults can be attributed to attentional (i.e. selective; Poliakoff, Ashworth, et al., 2006b) or other higher-level cognitive mechanisms (i.e. general cognitive slowing; cf. Bucur et al., 2005; Laurienti et al., 2006). Instead, it is likely that this is due to relatively poorer sensory organ functioning (Nusbaum, 1999) and/or slower afferent (and also efferent) conduction (Dorfman and Bosley, 1979; Rivner, Swift and Malik, 2001). It is less certain why some younger participants also show slower unisensory responses, given that sensory acuity/sensitivity is generally better in this group, but is likely to reflect individual variability within the population.

\section{Solutions and alternatives for estimating enhanced integration}

The current experiment has highlighted some fundamental issues with interpreting age-related differences in multisensory integration based on unisensory performance, such as with the test of the race model. However, we acknowledge that this apparent enhancement of multisensory integration in older adults cannot be completely rejected as being due to a floor/ceiling effect, and it is possible that the results are a genuine reflection of the principle of inverse effectiveness in older adults. In order to dissociate these two effects, Holmes (2009a, 2009b) has proposed a number of a priori experimental manipulations. For example, performance could be constrained to an intermediate level for each individual prior to testing, such as by decreasing stimulus intensity or saliency. In this case, all participants would fall within a set performance criteria, and so avoid

517 extreme responses. Similarly, the analysis could also be constrained/transformed post hoc (Laurienti et al., 2006), such that extreme values are excluded from the data set.

A further solution could be to avoid using experimental paradigms where performance is likely to be more variable within one of the participant groups, where this could provide more opportunity for enhanced integration (see "Variability Rule"; Otto, Dassy and Mamassian, 2013). Previous RT studies 
have reported a larger time window of integration in older adults, plus more variability in older group responses (Diederich, Colonius and Schomburg, 2008; Hugenschmidt, Mozolic and Laurienti, 2009). Similarly, the overall mean and standard deviation of older adults in the current investigation $(\mathrm{mean}=359 \mathrm{~ms}, \mathrm{SD}=85 \mathrm{~ms})$ was higher than young adults $(\mathrm{mean}=334 \mathrm{~ms}, \mathrm{SD}=65 \mathrm{~ms})$, and can be observed by a larger spread of the data (Table 1). We attempted to account for this large variability in participants' reaction times by measuring race model violation at percentiles rather than using time (ms) bins, therefore equating the distribution of RTs where race model violation occurs for all participants (cf. Laurienti et al., 2006). Stevenson and colleagues (2012) have also shown individual differences in MSI in non-RT based measures, even in a relatively more homogenous young adult group. Recent experiments within our lab have attempted to reduce this within and between group variability by initially constraining individual unisensory performance, thus enabling the influence of multisensory stimulation to be more easily observed (Poole et al., 2015a, 2015b; Couth, Gowen and Poliakoff, 2016). Similarly, there are other studies which have used different experimental approaches and models of multisensory processing which have demonstrated age-related differences, such as differences in sensory (re)weighting using (e.g. Jeka, Allison and Kiemel, 2010), as well as selective attention (e.g. Poliakoff, Ashworth, et al., 2006) and temporal processing (Poliakoff, Shore, et al., 2006).

It is also important to note that there are aspects of our methods which may differ to previous studies which have also used a simple RT paradigm, such as side of stimulation (e.g. unilateral vs. bilateral; Mahoney et al., 2011), stimulus type and intensity (e.g. bone conduction vs. electrical impulses; Mahoney et al., 2011), and response type (e.g. foot press vs. finger press; Peiffer et al., 2007) which could have influenced the magnitude of race model violation compared to other studies

544 (see also McGovern et al., 2014 for task specific effects on multisensory processing in ageing). Additionally, there are aspects of our data analysis approach which might differ to previous studies using the test of the race model. For example, it has been suggested previously that the test of the 
not, could be indicative of coactivation (Eriksen et al., 1989; Mordkoff and Yantis, 1991). Accordingly, constraining the analysis to $10-25$ th percentiles, verifying race model violation by significant t-test results at each percentile, and using Bonferroni corrections may have been overly stringent in the current experiment, where there may have been more race model violation than reported. Nevertheless, while these methodological differences may have affected the race model violation observed in the current study, they cannot account for the relationship that we have observed between the magnitude of race model violation and unisensory RTs.

Finally, we are not claiming that the older adults in the current (or any previous) study were not integrating. The point that we are emphasising is that by using a simple RT paradigm and the test of the race model, it may give the appearance of enhanced integration in older adults compared to young, even if this is not the case. Furthermore, we are not suggesting that older adults do not receive response benefits from multisensory stimulation. In fact, older individuals with the slowest unisensory reaction times benefitted the most from multisensory stimulation, which could be useful for improving reactions and orientation towards external events in the environment, and for coordinating behaviour effectively. For example, Mahoney and colleagues (2014) have suggested visuotactile cross-walk stimulators to improve older adults' responses to external cues, whilst other groups have also suggested training exercises to improve the efficiency of MSI to aid perception (Mozolic et al., 2011; Setti et al., 2014) and balance behaviour (Hu and Woollacott, 1994a, 1994b).

\section{Conclusions}

Previous studies measuring response times have shown that older adults demonstrate a greater magnitude of race model violation compared to young adults. This has been interpreted as an enhancement of MSI in older adults. Here, we show that enhanced integration may have previously been driven by a subset of individuals within each age group. Moreover, we suggest that the test of the race model gives the impression of enhanced integration in older adults, but may instead reflect 

responses.

575 This work was supported by the Frederick Craven Moore Studentship Award; The University of Manchester.

\section{References}

Barrett, M. M. and Newell, F. N. (2015) 'Task-Specific, Age Related Effects in the Cross-Modal Identification and Localisation of Objects', Multisensory Research, 28(1-2), pp. 111-151. doi: 10.1163/22134808-00002479. coactivation in bimodal detection: evidence for the preservation of coactive processing in older adults.', The journals of gerontology. Series B, Psychological sciences and social sciences, 60(5), pp. P279-82. doi: 10.1093/geronb/60.5.P279. Hearing, 23(5), pp. 439-449. doi: 00003446-200210000-00006. 295X.113.1.148. P., Dale, W., McClintock, M. K. and Pinto, J. M. (2016) 'Global Sensory Impairment in Older Adults in the United States', Journal of the American Geriatrics Society, 64(2), pp. 306-313. doi: 10.1111/jgs.13955. 
Couth, S., Gowen, E. and Poliakoff, E. (2016) 'Investigating the spatial and temporal modulation of 597 visuotactile interactions in older adults', Experimental Brain Research, 234(5), pp. 1233-1248. doi: 10.1007/s00221-015-4431-5.

Diederich, A. and Colonius, H. (2004) 'Bimodal and trimodal multisensory enhancement: effects of stimulus onset and intensity on reaction time.', Perception \& Psychophysics, 66(8), pp. 1388-1404. doi: 10.3758/BF03195006.

602

Diederich, A., Colonius, H. and Schomburg, A. (2008) 'Assessing age-related multisensory enhancement with the time-window-of-integration model.', Neuropsychologia, 46(10), pp. 25562562. doi: 10.1016/j.neuropsychologia.2008.03.026.

Dorfman, L. J. and Bosley, T. M. (1979) ‘Age-related changes in peripheral and central nerve conduction in man.', Neurology, 29(1), pp. 38-44. doi: 10.1212/WNL.29.1.38.

Dumas, K., Holtzer, R. and Mahoney, J. R. (2016) 'Visual-Somatosensory Integration in Older Adults: Links to Sensory Functioning', Multisensory Research, 29(4-5), pp. 397-420. doi: 10.1163/2213480800002521. coactivation, divided attention, or what?', Perception \& psychophysics, 45(4), pp. 356-70. doi: 10.3758/BF03204950. Falkenstein, I. A., Cochran, D. E., Azen, S. P., Dustin, L., Tammewar, A. M., Kozak, I. and Freeman, W. R. (2008) 'Comparison of visual acuity in macular degeneration patients measured with snellen and early treatment diabetic retinopathy study charts.', Ophthalmology, 115(2), pp. 319-23. doi: 10.1016/j.ophtha.2007.05.028. 
grading the cognitive state of patients for the clinician.', Journal of psychiatric research, 12(3), pp. 189-98. doi: 10.1016/0022-3956(75)90026-6.

Hecht, D., Reiner, M. and Karni, A. (2008a) 'Enhancement of response times to bi- and tri-modal sensory stimuli during active movements.', Experimental Brain Research, 185(4), pp. 655-65. doi: 10.1007/s00221-007-1191-x.

Hecht, D., Reiner, M. and Karni, A. (2008b) 'Multisensory enhancement: gains in choice and in simple response times.', Experimental Brain Research, 189(2), pp. 133-43. doi: 10.1007/s00221-008-1410-0.

Holmes, N. P. (2009) 'Inverse effectiveness, multisensory integration, and the bodily self: some statistical considerations.', Consciousness and cognition, 18(3), pp. 762-765. doi: 10.1016/j.concog.2009.04.009.

Holmes, N. P. (2009) 'The principle of inverse effectiveness in multisensory integration: some statistical considerations.', Brain Topography, 21(3-4), pp. 168-176. doi: 10.1007/s10548-009-00972.

Hsiao, S. (1998) 'Similarities between touch and vision', in Morley, J. (ed.) Neural Aspects of Tactile Sensation (Advances in Psychology). 1st Editio. Amsterdam: El, pp. 131-165.

Hu, M. H. and Woollacott, M. H. (1994a) 'Multisensory training of standing balance in older adults: I. Postural stability and one-leg stance balance.', Journal of gerontology, 49(2), pp. M52-61. doi: 10.1093/geronj/49.2.M52.

Hu, M. H. and Woollacott, M. H. (1994b) 'Multisensory training of standing balance in older adults: II. Kinematic and electromyographic postural responses.', Journal of gerontology, 49(2), pp. M62-71. doi: 10.1093/geronj/49.2.M62.

Hugenschmidt, C., Mozolic, J. and Laurienti, P. (2009) 'Suppression of multisensory integration by modality-specific attention in aging', Neuroreport, 20(4), pp. 349-353. doi: 
Jeka, J. J., Allison, L. K. and Kiemel, T. (2010) 'The dynamics of visual reweighting in healthy and fall-

643 prone older adults.', Journal of motor behavior, 42(4), pp. 197-208. doi:

$644 \quad 10.1080 / 00222895.2010 .481693$.

645 Kaiser, P. K. (2009) 'Prospective evaluation of visual acuity assessment: a comparison of snellen

646 versus ETDRS charts in clinical practice (An AOS Thesis).', Transactions of the American

647 Ophthalmological Society, 107, pp. 311-24.

Kiesel, A., Miller, J. and Ulrich, R. (2007) 'Systematic biases and Type I error accumulation in tests of the race model inequality.', Behavior research methods, 39(3), pp. 539-51. doi:

650 10.3758/BF03193024.

Laurienti, P. J., Burdette, J. H., Maldjian, J. A. and Wallace, M. T. (2006) ‘Enhanced multisensory integration in older adults.', Neurobiology of aging, 27(8), pp. 1155-1163. doi: 10.1016/j.neurobiolaging.2005.05.024.

Lee, J., Poliakoff, E. and Spence, C. (2009) 'The effect of multimodal feedback presented via a touch screen on the performance of older adults', Haptic and Audio Interaction Design, 5763, pp. 128-135. doi: 10.1007/978-3-642-04076-4_14. Physical Activity Level in Older Adults', Multisensory research, 28. doi: 10.1163/22134808-00002470. evidence for psychophysical integrative differences in aging.', Multisensory research, 27(1), pp. 1742. doi: $10.1163 / 22134808-00002444$. across the senses in young and old adults.', Brain research, 1426, pp. 43-53. doi: 
665

666

667

668

669

670

671

672

673

674

675

676

677

678

679

680

681

682

683

684

685

686

Mahoney, J. R., Wang, C., Dumas, K. and Holtzer, R. (2014) 'Visual-somatosensory integration in aging: Does stimulus location really matter?', Visual neuroscience, 31(3), pp. 275-283. doi: $10.1017 /$ S0952523814000078.

McGovern, D. Roudaia, E., Stapleton, J., McGinnity, T. M. and Newell, F. N. (2014) 'The soundinduced flash illusion reveals dissociable age-related effects in multi sensory integration.', Frontiers in Aging Neuroscience, 6. doi: 10.3389/fnagi.2014.00250.

Meredith, M. A. and Stein, B. E. (1986) 'Visual, auditory, and somatosensory convergence on cells in superior colliculus results in multisensory integration.', Journal of neurophysiology, 56, pp. 640-662.

Miller, J. (1982) 'Divided attention: evidence for coactivation with redundant signals.', Cognitive psychology, 14(2), pp. 247-279. doi: 10.1016/0010-0285(82)90010-X.

Miller, J. and Ulrich, R. (2003) 'Simple reaction time and statistical facilitation: A parallel grains model', Cognitive Psychology, 46(2), pp. 101-151. doi: 10.1016/S0010-0285(02)00517-0.

Mordkoff, J. T. and Yantis, S. (1991) 'An interactive race model of divided attention.', Journal of experimental psychology. Human perception and performance, 17(2), pp. 520-538. doi: 10.1037/0096-1523.17.2.520.

Mozolic, J. L., Long, A. B., Morgan, A. R., Rawley-Payne, M. and Laurienti, P. J. (2011) ‘A cognitive training intervention improves modality-specific attention in a randomized controlled trial of healthy older adults.', Neurobiology of aging, 32(4), pp. 655-68. doi: 10.1016/j.neurobiolaging.2009.04.013.

Nickerson, R. S. (1973) 'Intersensory facilitation of reaction time: energy summation or preparation enhancement?', Psychological review, 80(6), pp. 489-509. doi: 10.1037/h0035437. 
Oldfield, R. C. (1971) 'The assessment and analysis of handedness: The Edinburgh inventory', Neuropsychologia, 9(1), pp. 97-113. doi: 10.1016/0028-3932(71)90067-4.

Otto, T. U., Dassy, B. and Mamassian, P. (2013) 'Principles of multisensory behavior.', The Journal of neuroscience : the official journal of the Society for Neuroscience, 33(17), pp. 7463-74. doi: 10.1523/JNEUROSCI.4678-12.2013.

Peiffer, A. M., Mozolic, J. L., Hugenschmidt, C. E. and Laurienti, P. J. (2007) ‘Age-related multisensory enhancement in a simple audiovisual detection task.', Neuroreport, 18(10), pp. 1077-81. doi: 10.1097/WNR.0b013e3281e72ae7.

Poliakoff, E., Ashworth, S., Lowe, C. and Spence, C. (2006) 'Vision and touch in ageing: crossmodal 696 selective attention and visuotactile spatial interactions.', Neuropsychologia, 44(4), pp. 507-17. doi: 10.1016/j.neuropsychologia.2005.07.004.

Poliakoff, E., Shore, D. I., Lowe, C. and Spence, C. (2006) 'Visuotactile temporal order judgments in ageing', Neuroscience Letters, 396, pp. 207-211. doi: 10.1016/j.neulet.2005.11.034. congruency task for measuring the limits of visual-tactile interactions within and between groups', Multisensory research. doi: 10.1163/22134808-00002475.

Poole, D., Gowen, E., Warren, P. A. and Poliakoff, E. (2015) 'Investigating Visual-Tactile Interactions over Time and Space in Adults with Autism.', Journal of autism and developmental disorders. doi: 10.1007/s10803-015-2492-8.

Raab, D. H. (1962) 'Statistical facilitation of simple reaction times', Transactions of the New York Academy of Sciences, 24(5 Series II), pp. 574-590. doi: 10.1111/j.2164-0947.1962.tb01433.x. 
710

711

712

713

714

715

716

717

718

719

720

721

722

723

724

725

726

727

728

729

730

731

van Selst, M. and Jolicoeur, P. (1994) 'A solution to the effect of sample size on outlier elimination', The Quarterly Journal of Experimental Psychology Section A, 47, pp. 631-650. doi: $10.1080 / 14640749408401131$.

Setti, A., Stapleton, J., Leahy, D., Walsh, C., Kenny, R. A. and Newell, F. N. (2014) 'Improving the efficiency of multisensory integration in older adults: audio-visual temporal discrimination training reduces susceptibility to the sound-induced flash illusion.', Neuropsychologia, 61, pp. 259-68. doi: 10.1016/j.neuropsychologia.2014.06.027.

Stein, B. E. and Meredith, M. A. (1993) The Merging of the Senses. MIT Press.

Stevenson, R. A., Zemtsov, R. K. and Wallace, M. T. (2012) 'Individual differences in the multisensory temporal binding window predict susceptibility to audiovisual illusions.', Journal of experimental psychology. Human perception and performance, 38(6), pp. 1517-29. doi: 10.1037/a0027339.

Sullivan, E. V, Adalsteinsson, E., Hedehus, M., Ju, C., Moseley, M., Lim, K. O. and Pfefferbaum, A. (2001) 'Equivalent disruption of regional white matter microstructure in ageing healthy men and women.', Neuroreport, 12(1), pp. 99-104. doi: 10.1097/00001756-200101220-00027.

Teramoto, W., Honda, K., Furuta, K. and Sekiyama, K. (2017) 'Visuotactile interaction even in far sagittal space in older adults with decreased gait and balance functions', Experimental Brain Research, pp. 1-15. doi: 10.1007/s00221-017-4975-7.

Ulrich, R., Miller, J. and Schröter, H. (2007) 'Testing the race model inequality: an algorithm and computer programs.', Behavior research methods, 39(2), pp. 291-302. doi: 10.3758/BF03193160. 


\section{Figure captions}

Figure 1. Stimulus delivery apparatus. Participants used their dominant hand for tactile stimulation. Vision of the stimulated finger was occluded by the insulated casing.

Figure 2. CDFs for each bimodal (top three panels) and the trimodal (bottom panel) stimulus combinations. Young adults (left panels) and older adults (right panels) are displayed separately.

Figure 3. Probability difference curves for bimodal (top three panels) and trimodal (bottom panel) stimuli. Blue asterisks represent significant race model violation for older adults, whereas red crosses represent significant race model violation for young adults ( $p<.0125$, Bonferroni correction).

Figure 4. Probability difference curves for bimodal (top three panels) and trimodal (bottom panel) stimuli for RMv and RMa sub-groups. Blue asterisks represent significant race model violation for the older RMv group, red crosses represent significant race model violation for the young RMv group, and double black asterisks represent a significant difference between young and older RMv groups ( $p<.0125$, Bonferroni correction).

Figure $5 . \quad$ a) Mean fastest unisensory RTs for the VA stimulus combination for the older and young RMv sub-group (light bars) and RMa sub-group (dark bars). Single asterisks represent significant differences between RMv and RMa sub-groups, while double asterisks represent significant differences between young and older adults within the RMv sub-group. Scatter plots show correlations between the magnitude of race model violation and the fastest unisensory RT for b) older (blue diamonds) and c) young (red squares) participants. Positive and negative data points correspond to participants within the RMv and RMa sub-groups, respectively. Crossed data points were removed from the analysis. 


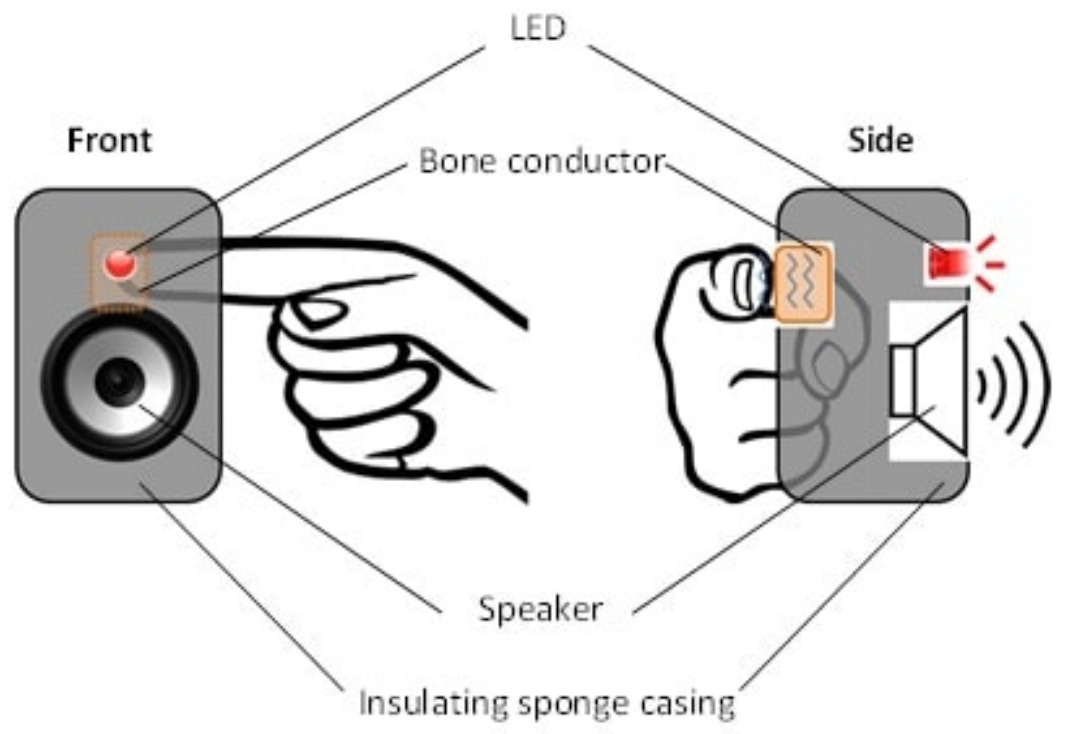



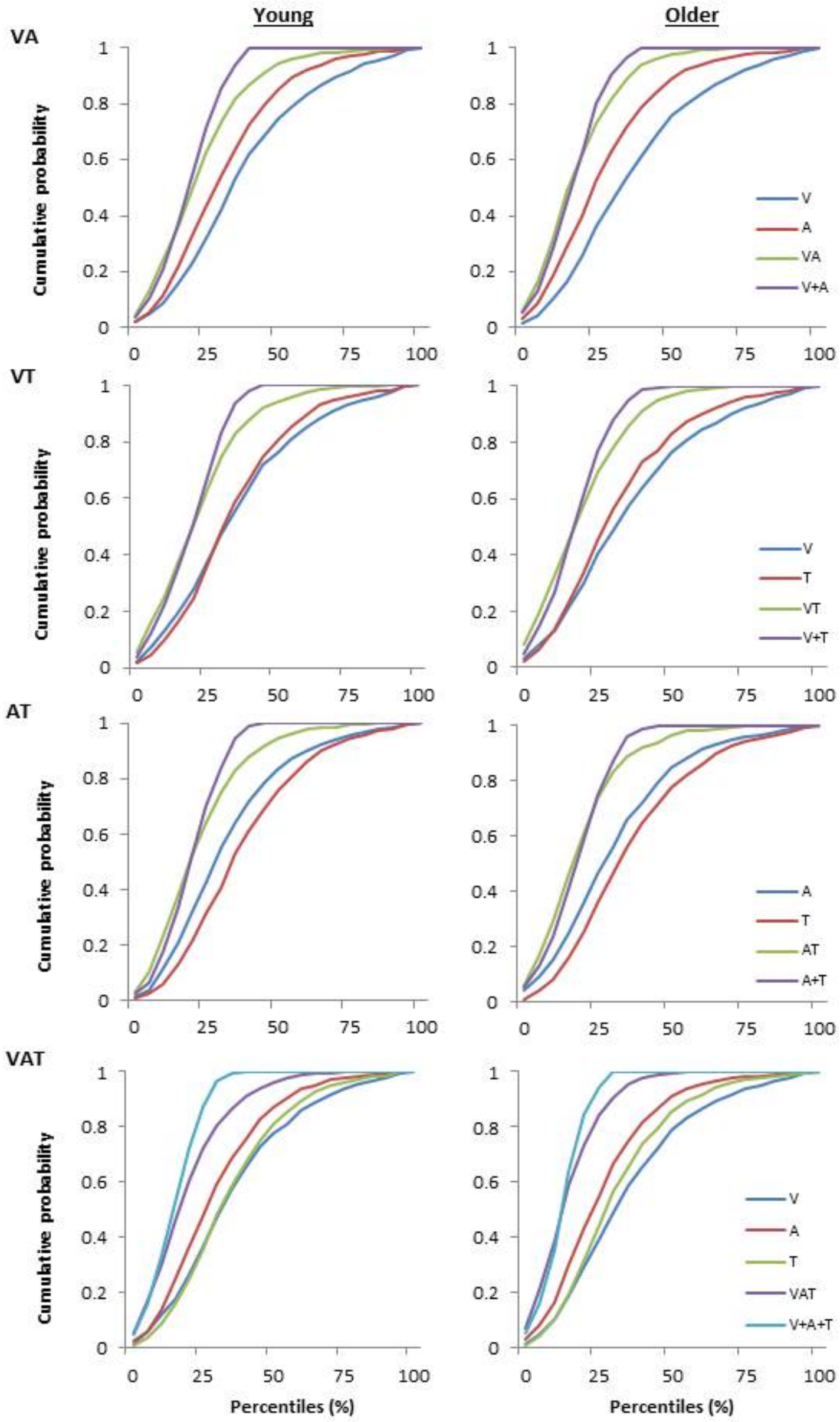

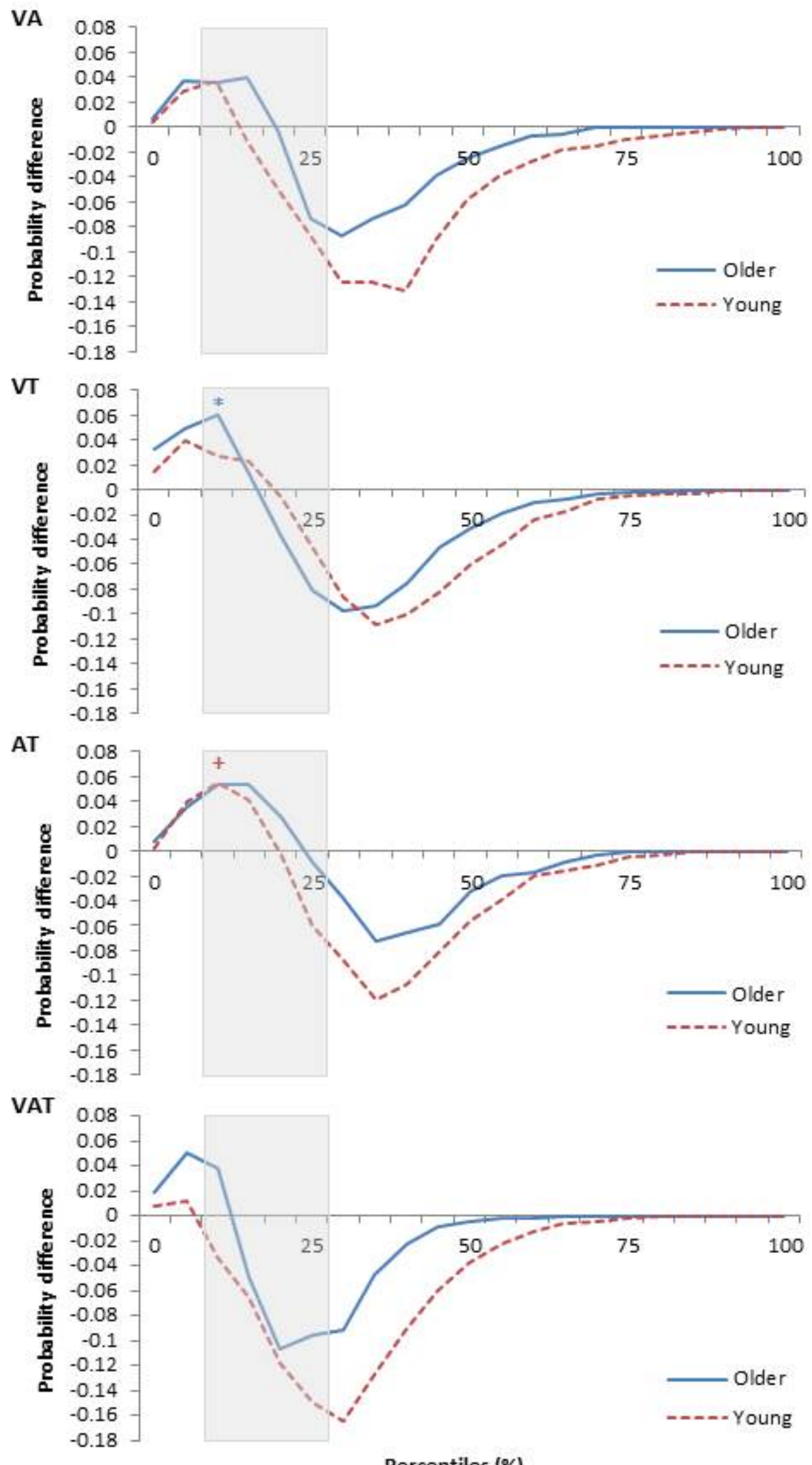

Percentiles (\%) 
VA
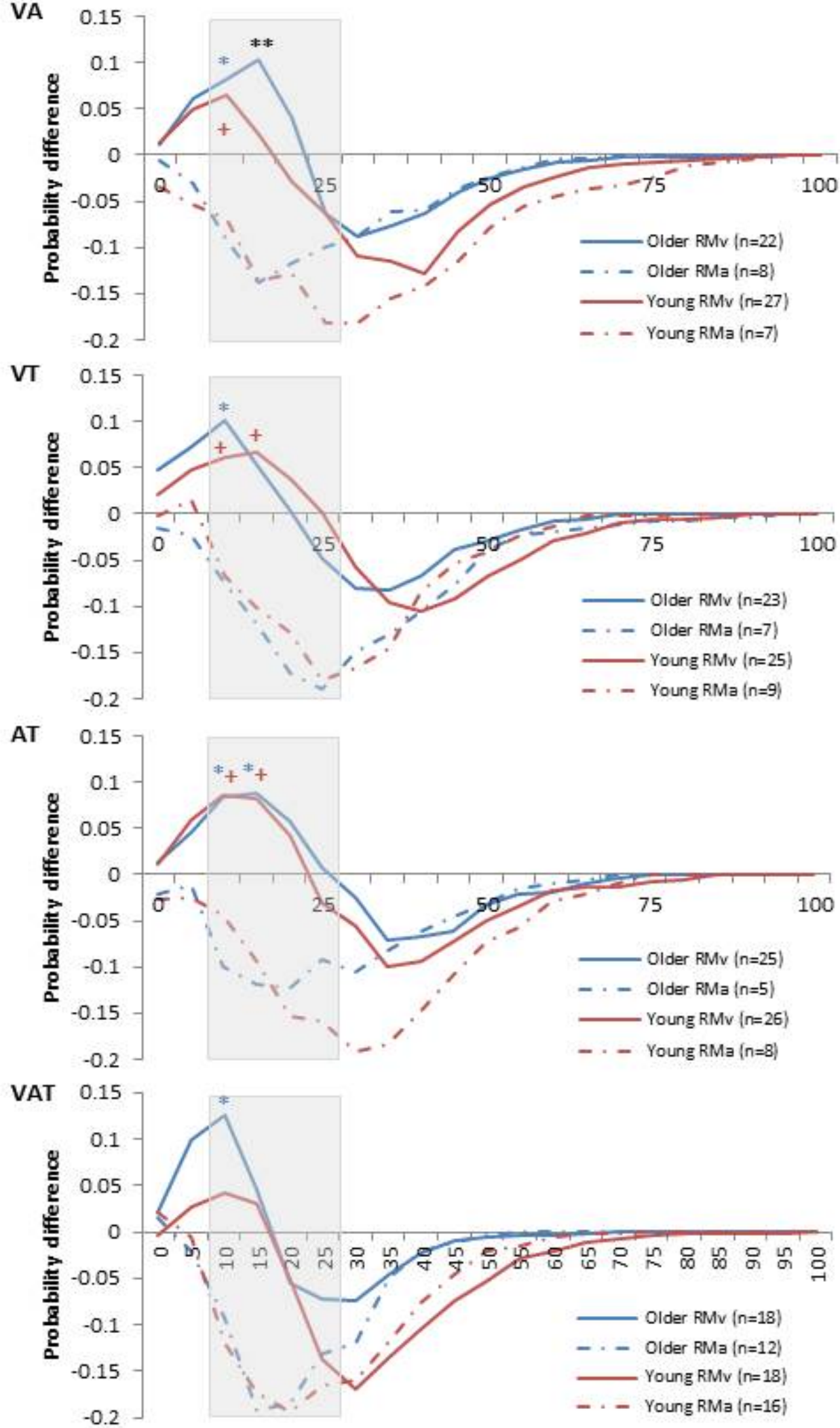

Percentiles 

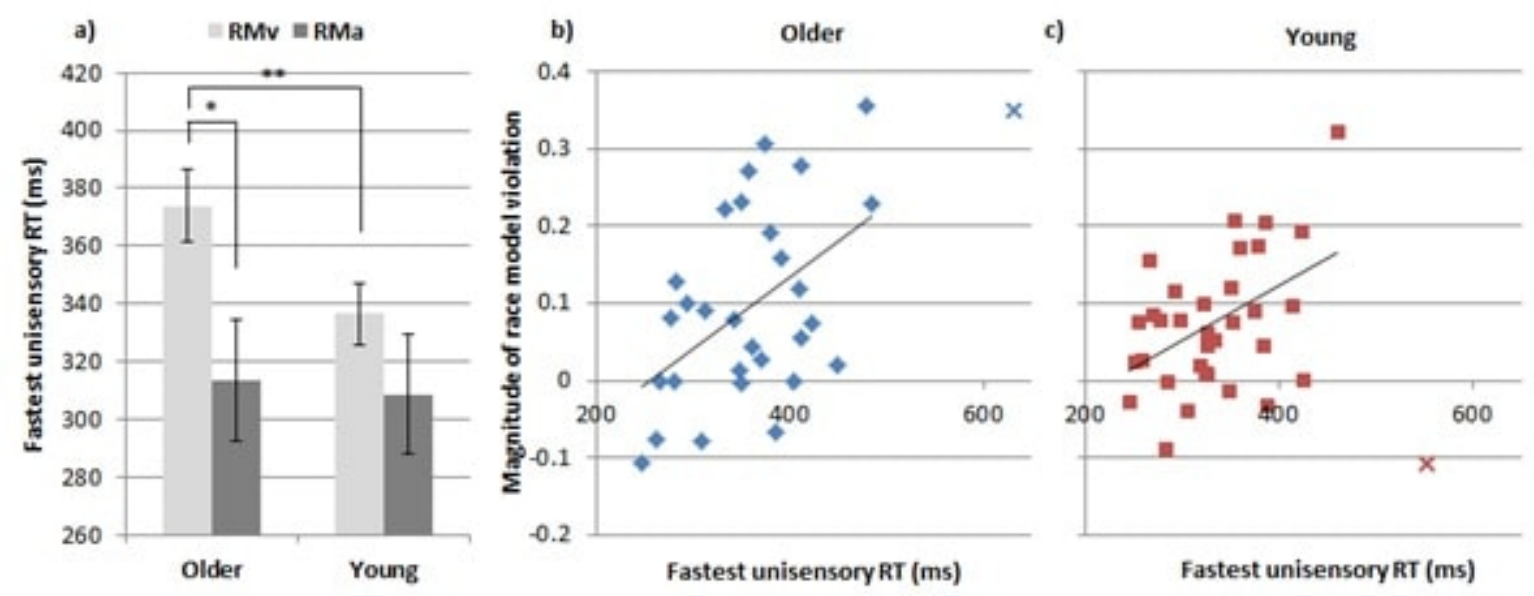